\title{
27. ICHTHYOLITHS FROM SITE 436, NORTHWEST PACIFIC, LEG 56, DEEP SEA DRILLING PROJECT
}

\author{
Patricia S. Doyle and William R. Riedel, Scripps Institution of Oceanography, La Jolla, California
}

\section{MATERIAL EXAMINED}

Approximately 20 meters of the section near the bottom of Hole 436 was "unfossiliferous," overlain by middle Miocene mudstone with siliceous microfossils and underlain by Cretaceous chert. Therefore we attempt to determine the age of these pelagic clays on the basis of the ichthyoliths. Site 436 is at $39^{\circ} 55.96^{\prime} \mathrm{N}$, $145^{\circ} 33.47^{\prime} \mathrm{E}$, in a water depth of 5240 meters. The cores investigated here (Nos. 39 and 40) are from 359.5-378 meters below the sea floor. In addition, we studied one sample from Core 41, containing Late Cretaceous radiolarians $(436-41-1,26-31 \mathrm{~cm})$.

Our system of discrimination and nomenclature is that developed by Doyle et al. (1974, pp. 825-905, pls. $1 \mathrm{~A}-\mathrm{J}, 2 \mathrm{~A}-\mathrm{M}, 3,4)$ and modified in subsequent papers (Dunsworth et al., 1975, pp. 853-863, pls. 1, 2; Ramsey et al., 1976, pp. 129-142, pls. 1-4; Edgerton et al., 1977, pp. 194-205, pl. 1; Doyle et al., 1978, pp. 743-759, pls. 1-4).

\section{RESULTS}

The ichthyoliths occurring in the Cretaceous sample (436-41-1, 26-31 cm) are illustrated in Plate 1, and two of them that show promise for stratigraphic application are described as new subtypes.

All ichthyoliths previously described have been looked for in the Site 436 samples (except for Triangle complex transverse line, which appears erratically throughout the Tertiary), and the numbers of specimens of each subtype found are recorded in Table 1. In the table the ichthyoliths are arranged in the order of their earliest occurrence in Giant Piston Core 3 (GPC-3), collected by C. D. Hollister at $30^{\circ} 20^{\prime} \mathrm{N}, 157^{\circ} 49^{\prime} \mathrm{W}$ (Doyle and Riedel, in press, pls. 1-9) because of the suitability of that large-diameter core as a standard for ichthyolith stratigraphy. Figure 1 shows the numbers of triangular $(a 8,9)$ ichthyoliths per gram of dry sediment in the samples from Site 436, and Figure 2 shows the concentration of the same type ichthyoliths in 24 samples from GPC-3.

That ichthyoliths in the Site 436 material are not very well preserved is evident in the high proportion of unidentifiable tooth-like fragments listed in the column "Total number of triangular $(a 8,9)$ ichthyoliths" to the numbers listed in the main body of Table 1. Also, delicate, scale-like ichthyoliths are rare, probably because of their greater susceptibility to solution or mechanical attrition.
Comparison of the order of earliest occurrences of the most reliable ichthyoliths (asterisked in Table 1) in the Site 436 sequence with that in GPC-3 suggests that the Oligocene/Miocene boundary occurs near the bottom of Core 436-39, the Eocene/Oligocene boundary near Section 5 of Core 436-40, and the Cretaceous/Eocene boundary between Cores $436-40$ and $436-41$. These are our conclusions despite the presence of several anomalies, which may be due to real differences in ichthyolith ranges between this area and those previously studied. The upper limits of Small triangle long striations, Rounded apex triangle, Triangle hooked margin, and Triangle with triangular projection are unusually low. We have no explanation for the occurrence of the single specimen of Short rectangular with striations in a sample interpreted as Oligocene.

That Sample 436-40, CC (25-39 cm) is not Paleocene or Cretaceous is indicated by the absence of subtypes Striated blunt triangle, Prominent polygon, Wide triangle projection, Triangle long inline, Short triangle bowed inline, Beveled triangle mid inline, Triangle curved base, and Triangle concave base. The low percentages of the subtypes Triangle pointed margin ends and Triangle with triangular projection in the 40, CC assemblage suggests that the sample is not early Eocene (Doyle and Riedel, in press, table 1, sample interval 1691-1708 cm).

An additional correlation level is suggested by the peak in ichthyolith abundance in Section 1 of Core 436-40 (Figure 1), which may correspond to the early Oligocene peak in GPC-3 (Figure 2). The Paleocene abundance peak in GPC-3 may occur between Cores 436-40 and 436-41, and in fact at this level the ichthyolith evidence indicates a hiatus representing the Paleocene.

To compare the rates of accumulation at the central North Pacific location of GPC-3 and the more northwesterly location of Site 436, we can take the earliest occurrence of Small triangle long striations (early Miocene) and the lowest occurrence of Eocene ichthyoliths, in the two sequences. In GPC-3, with an estimated rate of accumulation of 0.2 to $0.3 \mathrm{~m} / \mathrm{m}$.y., these two levels are separated by about 8 meters. At Site 436 they are separated by about 12 meters, indicating a rate of accumulation approximately 1.5 times as high.

\section{DESCRIPTIVE SECTION}

Table 2 lists, by numerically ordered name-descriptions, the ichthyolith subtypes identified at Site 436 . The description of two new ichthyoliths requires the following changes to the system of descriptors 
TABLE 1

Occurrences of Ich thyoliths at Site 436

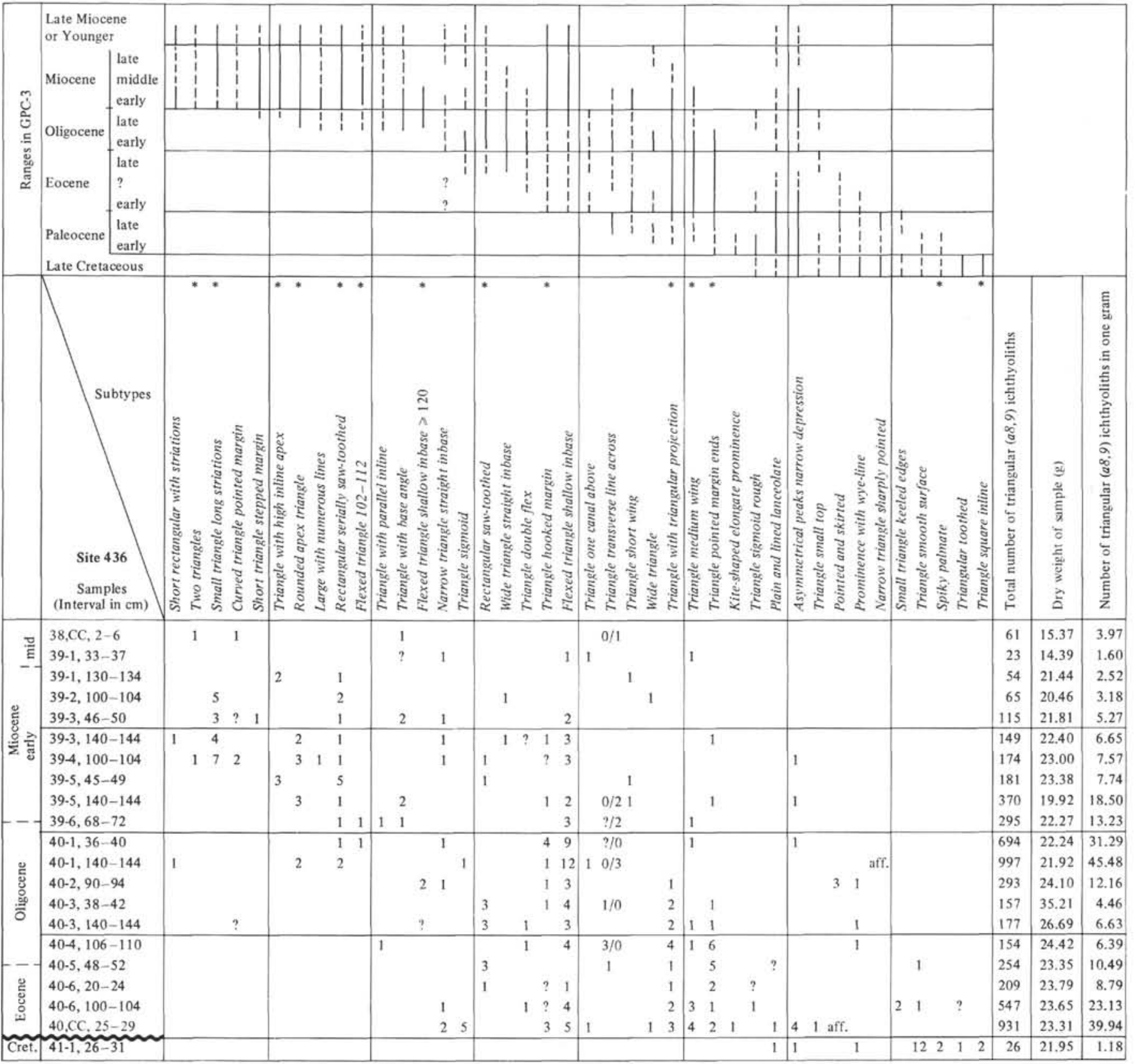

Note: Numerals in the body of the table refer to the number of specimens found. Question marks represent broken specimens in samples containing no other member of the subtype. Asterisks indicate the most reliable ichthyoliths. The ranges at the top of the table are those in GPC-3 (Doyle and Riedel, in press).

(Doyle et al., 1974; Dunsworth et al., 1975; Ramsey et al., 1976; Doyle et al., 1978):

To character $a$ (General outline), add the state

10. in a palmate, elliptical, or subcircular form, profusely dentate around almost the entire margin.

To character $p$ (Acuteness), add the states

4. truncate

5. asymmetrical

To character $q$ (General outline in apical view), add the state

5. not circular, acute on one side only

\section{New Subtypes}

$a 9 / b 1 / c 1,17,18 / d 1 / e 1 / f 1,5 / g 1 / h 2,3,4,5 / i 2,6,7 / j 2,3,4 /$ $k 5,8,9 / 10.20-0.80 / m 1.00-2.40 / n 9,10 / o 1 / p 1,4,5 / q 2,3$ Doyle and Riedel, new subtype.
Triangle smooth surface

(Plate 1, Figures 6-14)

Undescribed specimens of $a 9 / b 1,5$ Ramsey et al., 1976, pl. 4, figs. 13, 14. Undescribed forms, Doyle and Riedel, in press, pl. 8, figs. 24-29.

Description: Approximately triangular teeth with no transverse line. First margin unmodified, or coarsely saw-toothed on the lower half. Relative lengths of margins variable. First margin straight or concave, sometimes with most of the curvature basally. Second margin straight or convex, sometimes with most of the curvature basally. Inline arcuate, or more pointed and approximately parallel to outline, in some specimens closely approaching the outline at the base. Apex between sharp and blunt, or truncated, and often asymmetrical. General outline in apical view approximately circular, or not circular and obtuse laterally. 


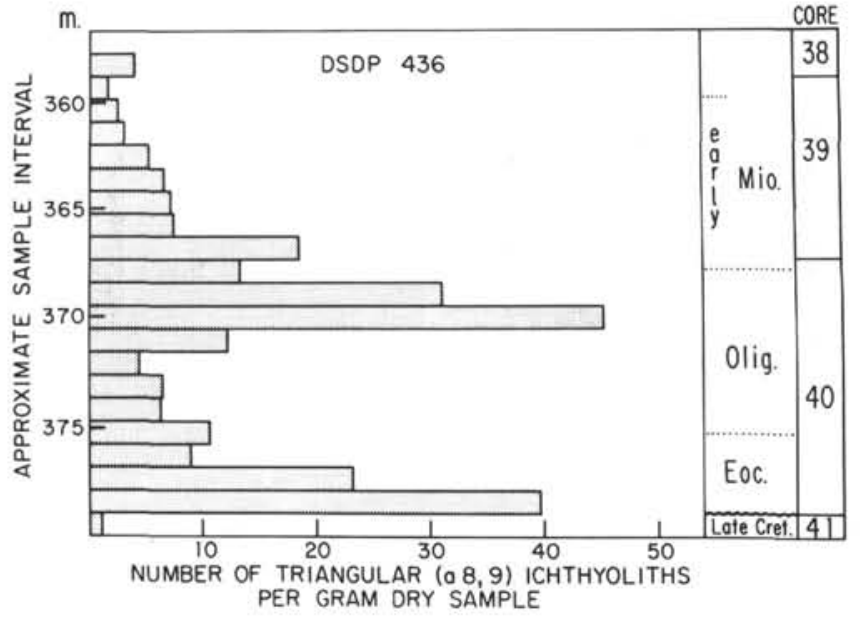

Figure 1. Concentrations of triangular ichthyoliths in samples examined from Site 436. Although the bars here and in Figure 2 adjoin one another, the samples are spaced approximately 1 meter apart. Ages determined by comparison with ichthyolith ranges in GPC-3.

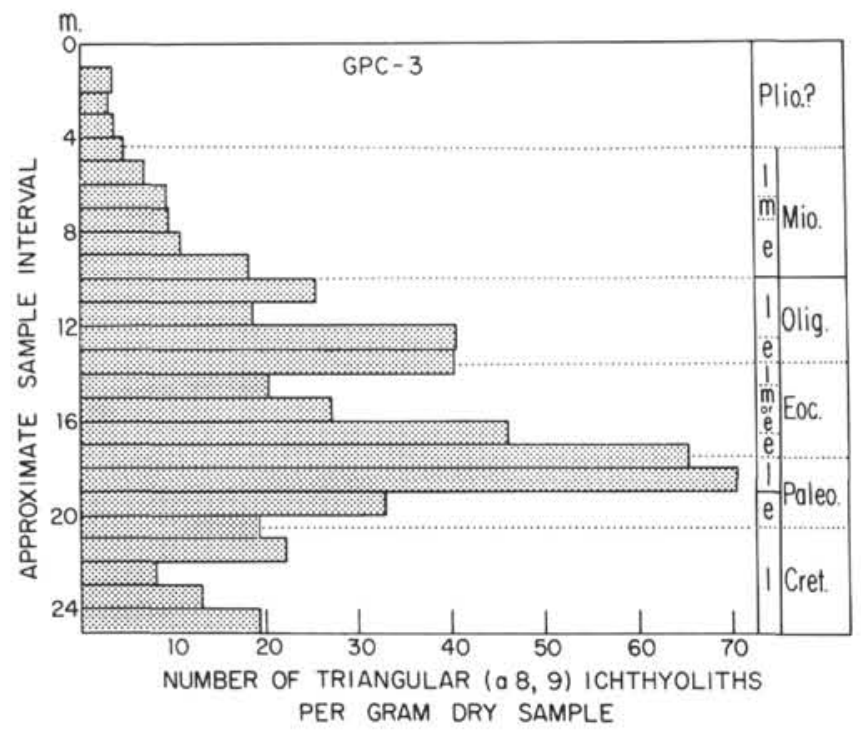

Figure 2. Concentrations of triangular ichthyoliths in samples examined from $\mathrm{GPC}-3$. The age determinations were made on the basis of ichthyoliths. Ages determined by ranges of ichthyoliths established in samples dated by other microfossils.
Remarks: A rather similar form (Doyle and Riedel, in press, pl. 2, figs. $3,4,6,8$ ) occurs in late Miocene or younger assemblages, which differs in the inline being usually more acute; nor have any of these younger specimens been observed with the coarse sawteeth on one margin.

$a 10 / b 1,2$ Doyle and Riedel, new type and subtype Spiky palmate

(Plate 1, Figures 1-5)

Undescribed forms, Doyle and Riedel, in press, pl. 8, figs. 8, 9.

Description: Palmate, elliptical, or subcircular forms, with the margin having many sharp to rounded projections of variable length around all but one end. Many specimens show subparallel, sometimes anastomosing lines over a large proportion of their area. In some, these lines radiate from the nondentate end.

\section{ACKNOWLEDGMENTS}

This research was supported partly by NSF Grant OCE7680884 and partly by the University of California. The task of typing was competently performed by Mary Hanger, and Carol Edgerton helped with the proofreading. John Van Couvering, editor of Micropaleontology, allowed us to use Figure 2 from a manuscript which is in press. Hans Thierstein (Scripps Institution of Oceanography) and Fritz Theyer (University of Hawaii) critically reviewed the manuscript and made suggestions resulting in its improvement.

\section{REFERENCES}

Doyle, P. S., Dunsworth, M. J., and Riedel, W. R., 1978. Ichthyoliths from some southeast Atlantic sediments. In Ryan, W. B. F., Bolli, H. M., et al., Init. Repts. DSDP, Suppl. to Vols. $38,39,40$, and 41: Washington (U.S. Govt. Printing Office), 743-759.

Doyle, P. S., Kennedy, G. G., and Riedel, W. R., 1974. Stratignathy. In Davies, T. A., Luyendyk, B. P., et al., Init. Repts. DSDP, 26: Washington (U.S. Govt. Printing Office).

Doyle, P. S., and Riedel, W. R. (in press). Cretaceous to Neogene ichthyoliths in a giant piston core from the central North Pacific. Micropaleontology.

Dunsworth, M. J., Doyle, P. S., and Riedel, W. R., 1975. Ichthyoliths from some NW Pacific Sediments, DSDP Leg 32. In Larson, R. L., Moberly, R., et al., Init. Repts. DSDP, 32: Washington (U.S. Govt. Printing Office), 853-863.

Edgerton, C. C., Doyle, P. S., and Riedel, W. R., 1977. Ichthyolith age determination of otherwise unfossiliferous Deep Sea Drilling Project cores. Micropaleontology, 23, 194-205.

Helms, P. B., and Riedel, W. R., 1971. Skeletal debris of fishes. In Winterer, E. L., et al., Init. Repts. DSDP, 7, pt. 2: Washington (U.S. Govt. Printing Office), 1709-1720.

Ramsey, C. A., Doyle, P. S., and Riedel, W. R., 1976. Ichthyoliths in late Mesozoic pelagic sediments, mainly from Italy. Micropaleontology, 22. 
TABLE 2

Name-Descriptions of Ichthyoliths in This Study (in numerical order, with equivalent colloquial names and authors)

\begin{tabular}{|c|c|c|c|c|c|c|c|c|c|c|c|c|}
\hline a & $\mathrm{b}$ & c & d & $\mathrm{e}$ & f & g & h & $\mathrm{i}$ & $\mathrm{j}$ & k & 1 & $\mathrm{~m}$ \\
\hline 3,4 & 1 & $1,2,3$ & $5,(4+5)$ & 2 & 2 & $1+8$ & 2 & & & & & \\
\hline 3,4 & 1,2 & $2,3,4$ & $1,2,3$ & 1 & $2+3$ & $1+8$ & 1 & & & & & \\
\hline 3 & 1 & 3 & 3,4 & 4 & 2 & $1+8$ & & & & & & \\
\hline 4 & 1,2 & 2 & $1,2,3$ & 2 & $1,(2+3)$ & $1+2$ & 1 & & & & & \\
\hline 5,6 & 3 & 2 & 1,2 & 1 & & & & & & & & \\
\hline 7 & 1 & 1 & 1 & 1 & & & & & & & & \\
\hline 7 & 1 & 2 & 3 & 3 & & & & & & & & \\
\hline 7 & 1 & 2 & 3 & 6 & & & & & & & & \\
\hline 7 & 6 & 3 & & & & & & & & & & \\
\hline 8 & 1,5 & 1 & 1 & $102-112$ & $26-36$ & & & & & & & \\
\hline 8 & 1,5 & 1 & 2 & $80-140$ & $26-36$ & & & & & & & \\
\hline+8 & 1,5 & 1 & 2,3 & $\geqslant 120$ & $\leqslant 25$ & & & & & & & \\
\hline 9 & 1 & 13 & 1 & 1 & $4+[1,(9+13+15)]$ & 1 & $2,3,4,5$ & 2 & 2 & 4,8 & $0.25-0.45$ & $2.0-3.0$ \\
\hline 8 & 1 & 2 & 2,3 & $60-100$ & $20-35$ & & & & & & & \\
\hline 9 & 1 & 1 & 1 & 1 & 1,4 & 1 & 1,2 & $2,6,8$ & $2,6,8$ & 2,3 & $<0.2$ & $1.5-2.0$ \\
\hline 9 & 1 & 1 & 1 & 1 & 1,2 & 1 & 1,2 & 2 & 2 & 2 & $0.2-0.4$ & $1.6-2.0$ \\
\hline 9 & 1 & 1 & 1 & 1 & 1 & 1 & $1,2,3$ & 2,3 & 2,3 & 2 & $0.25-0.45$ & $1.0-1.5$ \\
\hline 9 & 1 & 1 & 1 & 1 & 1,4 & 1 & 1 & 2 & 2 & 7 & $0.5-0.7$ & $2.5-3.5$ \\
\hline 9 & 1 & $1,17,18$ & 1 & 1 & 1,5 & 1 & $2,3,4,5$ & $2,6,7$ & $2,3,4$ & $5,8,9$ & $0.20-0.80$ & $1.00-2.40$ \\
\hline 9 & 1 & 1 & 1 & 1 & 1,8 & 1 & $3,4,5$ & 2,6 & 2,3 & 9 & 0 & $4.3-5.3$ \\
\hline 9 & 1 & 1 & 1 & 1 & 1 & 1 & $3,4,5$ & 3 & 3 & 8 & $0.2-0.8$ & $<1$ \\
\hline+9 & 1 & 1 & 1 & 1 & 1,3 & 1 & 5 & 9 & 9 & 8 & $0.05-0.25$ & $\geqslant 2.75$ \\
\hline 9 & 5 & 1 & 1 & 1 & 1 & 1 & 1 & 1 & 1 & 9 & 9 & 1,4 \\
\hline 9 & 1 & 1 & 1 & 1 & 2 & 1 & $1,2,3$ & 6 & 4 & 2,4 & $0.2-0.4$ & $1.0-2.0$ \\
\hline+9 & 1 & 1 & 1 & 1 & 4 & 1 & $2,3,4,5$ & 2,3 & 2,3 & 11 & 0 & 0 \\
\hline 8 & 1,5 & 1 & 3 & $80-125$ & $35-60$ & & & & & & & \\
\hline 9 & 1 & 1 & 1 & 1 & 4 & 1 & 5 & 6 & 2,6 & 5 & $0.5-0.75$ & $1.0-1.5$ \\
\hline+9 & 1 & 1 & 1 & 1 & 6 & 1 & 5 & 2 & 2 & 0.5 & $0.75-1.0$ & $1.5-1.8$ \\
\hline 9 & 5 & 1 & 1 & 1 & 1 & 1 & 1 & 8 & 1 & 2 & 2 & 2 \\
\hline 9 & 1 & 5 & 1 & 1 & $1,4,5$ & 1 & 1,3 & 2,3 & 6 & 2 & $<0.4$ & $1.5-2.0$ \\
\hline 9 & 1 & 6,7 & 1. & 1 & $1,4,5$ & 1 & 1,3 & 2,3 & 6 & 2 & $<0.4$ & $1.5-2.0$ \\
\hline+9 & 1 & 9,13 & 1 & 1 & $4+(6,7)$ & 1 & 4 & 2 & 2 & 7,8 & $0.2-0.6$ & $1.6-2.8$ \\
\hline 9 & 5 & 8,12 & 1 & 1 & 1 & 1 & 1 & $2+4$ & 1 & 2 & 2 & 2 \\
\hline 9 & 1 & 13 & 13 & 1 & 4 & 1 & 1 & 2 & 2 & 2 & $<0.6$ & $2.0-3.0$ \\
\hline+9 & 1 & 14 & 1 & 1 & $2+5$ & 1 & 2 & 4 & 2,3 & 2 & $0.1-0.4$ & $1.0-2.0$ \\
\hline 9 & 5 & 13 & 1 & 1 & 1 & 1 & 1 & $2+7$ & 1 & 2 & 4 & 3 \\
\hline 9 & 1 & 19 & 1,19 & 1 & 1 & 4 & $3,4,5$ & $2,3,10$ & $2,7,9$ & $0,8,9$ & 0 & $>2.0$ \\
\hline 9 & 5 & 1 & 1 & 1 & 1 & 1 & 1 & 1 & 2 & 2 & 2 & $1,2,3,4$ \\
\hline 9 & 5 & 1 & 1 & 1 & 1 & 1 & 1 & $1,3,5,6$ & 1 & 2,6 & 3 & 2,4 \\
\hline 9 & 5 & 1 & 1 & 1 & 1 & 1 & 1 & 1,6 & 3 & 1 & 1 & $2,3,5$ \\
\hline 9 & 5 & 1 & 1 & 1 & 1 & 1 & 2 & $4+(1,5)$ & 1 & 2 & 2 & 2 \\
\hline 9 & 5 & 1 & 1 & 1 & 1 & 2 & 2 & 4 & 1 & $2,4,6$ & 2,4 & 1 \\
\hline 9 & 5 & 1 & 1 & 3 & 1 & 1 & 1 & 1 & 1 & 2,7 & 2 & 2,4 \\
\hline 9 & 6 & $2,3,4,5$ & 2 & 1 & & & & & & & & \\
\hline 9 & 7 & & & & & & & & & & & \\
\hline 10 & 1,2 & & & & & & & & & & & \\
\hline
\end{tabular}


TABLE 2 - Continued

\begin{tabular}{|c|c|c|c|c|c|c|c|c|}
\hline \multirow[t]{12}{*}{$\mathrm{n}$} & o & $\mathrm{p}$ & q & r & s & $\mathrm{t}$ & u & \\
\hline & & & & & & & & Prominence with wye-line Doyle et al., 1978 \\
\hline & & & & & & & & Pointed and skirted Doyle et al., 1978 \\
\hline & & & & & & & & Kite-shaped elongate prominence Doyle et al., 1974 \\
\hline & & & & & & & & Plain and lined lanceolate Doyle et al., 1978 \\
\hline & & & & & & & & Large with numerous lines Doyle et al., 1974 \\
\hline & & & & & & & & Two triangles Doyle et al., 1974 \\
\hline & & & & & & & & Rectangular saw-toothed Doyle et al., 1974 \\
\hline & & & & & & & & Rectangular serially saw-toothed Dunsworth et al., 1975 \\
\hline & & & & & & & & Asymmetrical peaks narrow depression Doyle et al., 1974 \\
\hline & & & & & & & & Flexed triangle 102-112 Doyle et al., 1974 \\
\hline & & & & & & & & Flexed triangle shallow inbase Doyle et al., 1974 \\
\hline \multirow{2}{*}{$\underline{2,3,4,6,7}$} & 2 & 1 & 1 & & & & & $\begin{array}{l}\text { Flexed triangle shallow in base } \geqslant 120 \text { Dunsworth et al., 1974, } \\
\text { emend. Edgerton et a1., } 1977\end{array}$ \\
\hline & & & & & & & & Triangle double flex Dunsworth et al., 1975 \\
\hline 3,4 & 1 & 1,3 & & & & & & Triangle with high inline apex Doyle et al., 1974 \\
\hline 4,5 & 1 & 1,3 & & & & & & Narrow triangle straight inbase Doyle et al., 1974 \\
\hline 4,5 & 1 & 3 & & & & & & Wide triangle straight inbase Doyle et al., 1974 \\
\hline 2 & 1 & 1,2 & & & & & & Triangle with parallel inline Doyle et al., 1974 \\
\hline 9,10 & 1 & $1,4,5$ & 2,3 & & & & & Triangle smooth surface new subtype \\
\hline 9 & 1 & $\frac{1, \pi, 0}{2}$ & $\frac{1}{1}$ & & & & & Narrow triangle sharply pointed Ramsey et al., 1976 \\
\hline $4,5,6,7,8$ & 1 & 3 & & & & & & Wide triangle Dunsworth et al., 1975 \\
\hline$\frac{2}{2}$ & 1 & 2 & & & & & & Triangle sigmoid Dunsworth et al., 1975 , \\
\hline 1,3 & 9 & 3 & $0.05-0.25$ & $\geqslant 2.75$ & 0 & 2 & 1 & emend. Edgerton et al, 1977 \\
\hline $3,4,5$ & 2 & 1 & & & & & & Curved triangle pointed margin Doyle et al., 1974 \\
\hline $4,5,7,8$ & 1 & 3 & 1 & & & & & $\begin{array}{l}\text { Triangle square inline Doyle et al., in press, } \\
\text { emend. Edgerton et al., } 1977\end{array}$ \\
\hline 9 & 1 & 3 & 4 & & & & & Small triangle keeled edges Ramsey et al., 1976 \\
\hline 2 & 1 & 1 & & & & & & Small triangle long striations Dunsworth et al., 1975 \\
\hline 2 & 2 & 0,6 & $0.75-1.0$ & $1.5-1.8$ & 0 & 1 & 1 & \\
\hline 1 & 1 & 1 & & & & & & Triangle short wing Doyle et al., 1974 \\
\hline 1 & 1 & 1 & & & & & & Triangle medium wing Doyle et al., 1974 \\
\hline$\frac{1}{2}$ & 1,2 & 1 & & & & & & Triangle with base angle Dunswcrth et al., 1975 \\
\hline 2 & 9 & 3,8 & $0.2-0.6$ & $1.6-2.8$ & 0 & 1 & 1 & \\
\hline 3 & 3 & 2 & & & & & & Triangle pointed margin ends Doyle et al., 1974 \\
\hline 1 & 1 & 1 & & & & & & Triangle hooked margin Doyle et al., 1974 \\
\hline 1 & 1 & 3 & $<0.4$ & $1.0-2.0$ & 0 & 1 & & \\
\hline 9,10 & 1 & 1 & 2 & & & & & Triangle sigmoid rough Ramsey et al., 1976 \\
\hline $1,2,3,4$ & 2 & 2,3 & 0 & 0 & $<2.75$ & 1,2 & 1 & Triangle small top Ramsey et al., 1976 \\
\hline 2,3 & 3 & 3,8 & $>0.25$ & $<2$ & 0 & $\frac{1}{2}$ & & $\begin{array}{l}\text { Short triangle stepped margin Doyle et al., 1974, } \\
\text { emend. Edgerton et al., } 1977\end{array}$ \\
\hline $2,3,5$ & 3,4 & 2 & 0 & 0 & $\leqslant 1.0$ & 2,3 & & Short rectangular with striations Doyle et al., 1974 \\
\hline$\frac{2,0,2}{2}$ & 4 & 8 & $0.3-0.6$ & $1.0-2.5$ & 0 & 1 & & Triangle one canal above Doyle et al., 1976 \\
\hline 1 & 4 & 3 & $0.2-0.4$ & $1.5-2.5$ & 0 & 1 & & Triangle transverse line Doyle et al., 1974 \\
\hline 3 & 1,2 & 2,3 & 0 & $>1$ & $>3$ & 2 & & Triangle with triangular projection Doyle et al., 1974 \\
\hline & & & & & & & & Triangular-toothed Doyle et al., 1978 \\
\hline & & & & & & & & Rounded apex triangle Doyle et al., 1974 \\
\hline & & & & & & & & Spiky palmate new subtype \\
\hline
\end{tabular}




\section{PLATE 1}

All figures are magnified $\times 163$ and are from DSDP Sample 436-41-1, 26-31 cm, unless otherwise indicated. Letter-number combinations in the forms Sl.4 and M36 indicate slide numbers and England Finder positions, respectively, of the illustrated specimens.

Figures 1-5 a $\quad a 10 / b 1,2$ new subtype, Spiky palmate.

1. Sl.2, U11.

2. Sample 146-11-2, 1-3 cm, F2, L22. Late Cretaceous?

3. GPC-3, 2390-2400 cm, Sl.4, M38. Late Cretaceous.

4. Sample 385-13-2, 46-52 cm, Sl.1, 018. Maestrichtian.

5. GPC-3, 2103-2116 cm, Sl.7, H37. Late Cretaceous.

Figures 6-14 $a 9 / b 1 / c 1,17,18 / d 1 / e 1 / f 1,5 / g 1 / h 2,3,4,5 / i 2,6,7 / j 2,3,4 / k 5,8,9 /$ 10.20-0.80 / m1.00- 2.40 / n9,10 / ol / p1,4,5 / q2,3 new subtype, Triangle smooth surface.

6. GPC-3, 2290-2304 cm, S1.2, C25. × 106. Late Cretaceous.

7. GPC-3, $2250 \mathrm{~cm}, \mathrm{Sl} .1, \mathrm{~B} 15 . \times 106$. Late Cretaceous.

8. Sl.4, J20.

9. Sl.9, C33.

10. Sl.10, Q20.

11. Sl.12, O26.

12. Sl.4, J26.

13. Sl.8, R19.

14. S1.7, E22.

Figures 15, 16 Undescribed forms similar to Plain and lined lanceolate.

15. Sl.10, U13.

16. Sl.5, E26.

Figure $17 a 9 / b 6 / c 2,3,4,5 / d 2 / e 1$ Ramsey et al. (1976), Triangular toothed. S1.4, D36.

Figures 18, 19 Forms similar to those figured by Helms and Riedel (1971, pl. 2, figs. 20-25).

18. Sl.14, C24.

19. Sl.1, Q31.

Figure $20 a 3,4 / b 1 / c 1,2,3 / d 5,(4+5) / e 2 / f 2 / g 1+8 / h 2$ Doyle et al. (1978), Prominence with wye-line. Sl.13, C13.

Figure $21 \quad a 7 / b 6 / c 3$ Doyle et al. (1974), Asymmetrical peaks narrow depression. Sl.4, N45.

Figure 22 Form similar to Capped triangle, differing in that the length/width ratio of the portion below the transverse line is smaller. Sl.7, O25.

Figure $23 a 9 / b 1 / c l / d l / e l / f 9+(12,13)+4 / g 1 / h 4,5 / i 2,3 / j 2,3 / k 11 / l 0 / m 0.9-1.4 /$ $n 3,4,9 / o 1 / p 3 / q 1$ Doyle et al. (1974), Striated blunt triangle. Sl.12, L23.

Figure $24 \quad$ Undescribed form. Sl.8, D40.

Figure $25 \quad$ Form similar to Straight triangle keeled edges. Sl.13, G35.

Figures 26-28 Undescribed forms.

26. Sl.3, F34.

27. Sl.14, D26.

28. Sl.11, N39.

Figures 29, 30 Forms similar to Striated blunt triangle and undescribed forms illustrated from a Late Cretaceous assemblage by Doyle and Riedel (in press, pl. 9, figs. 12, 17, 18, and 26).

29. Sl.9, $\mathrm{H} 24$.

30. S1.5, S26. 
PLATE 1
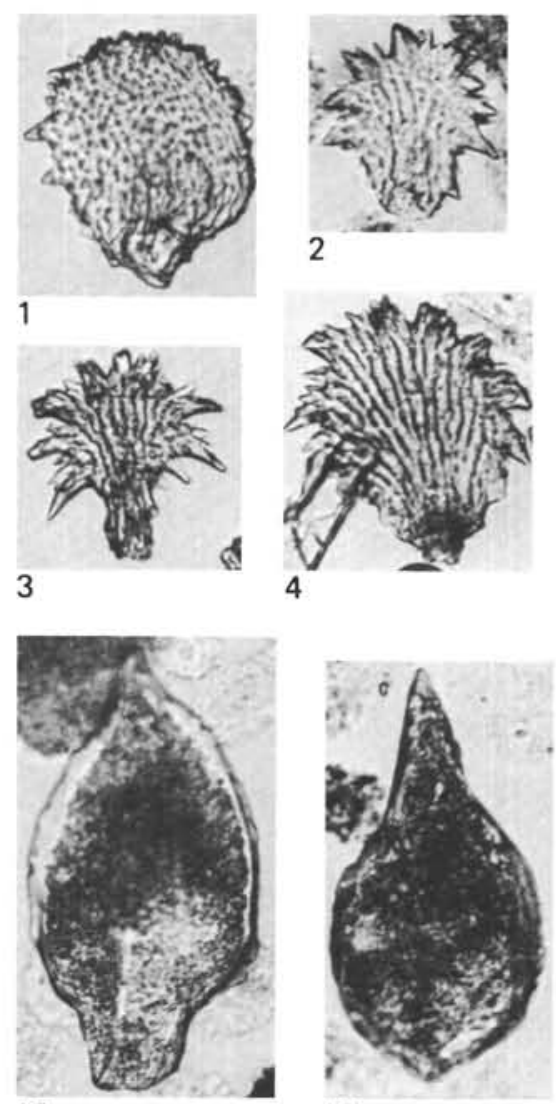

15
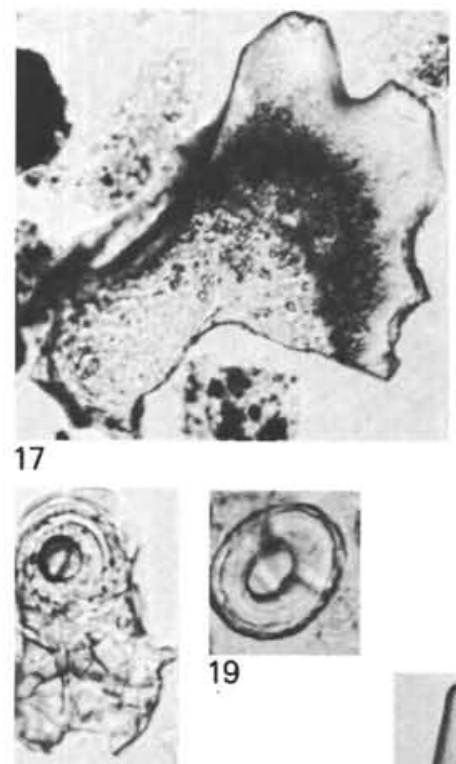

18

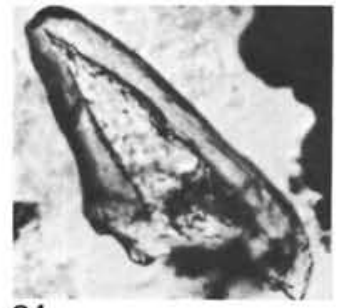

24

20

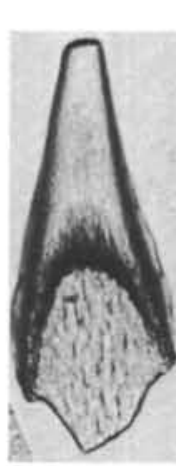

25
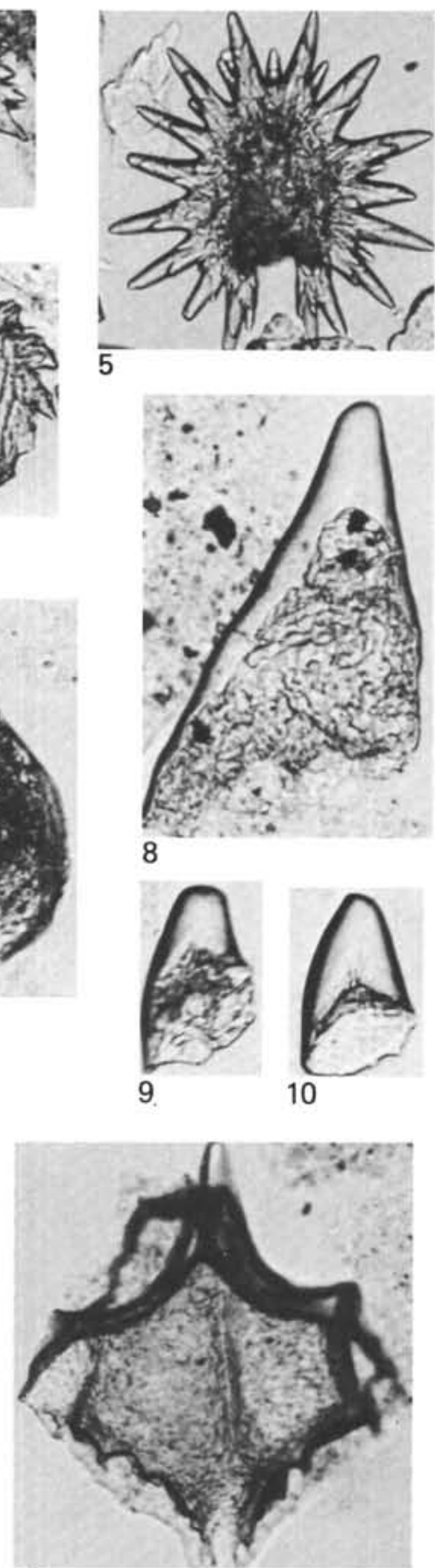

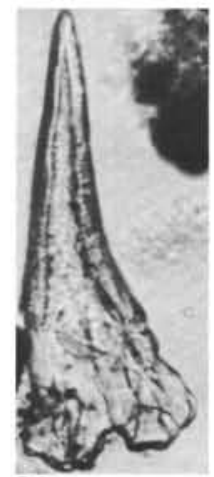

26
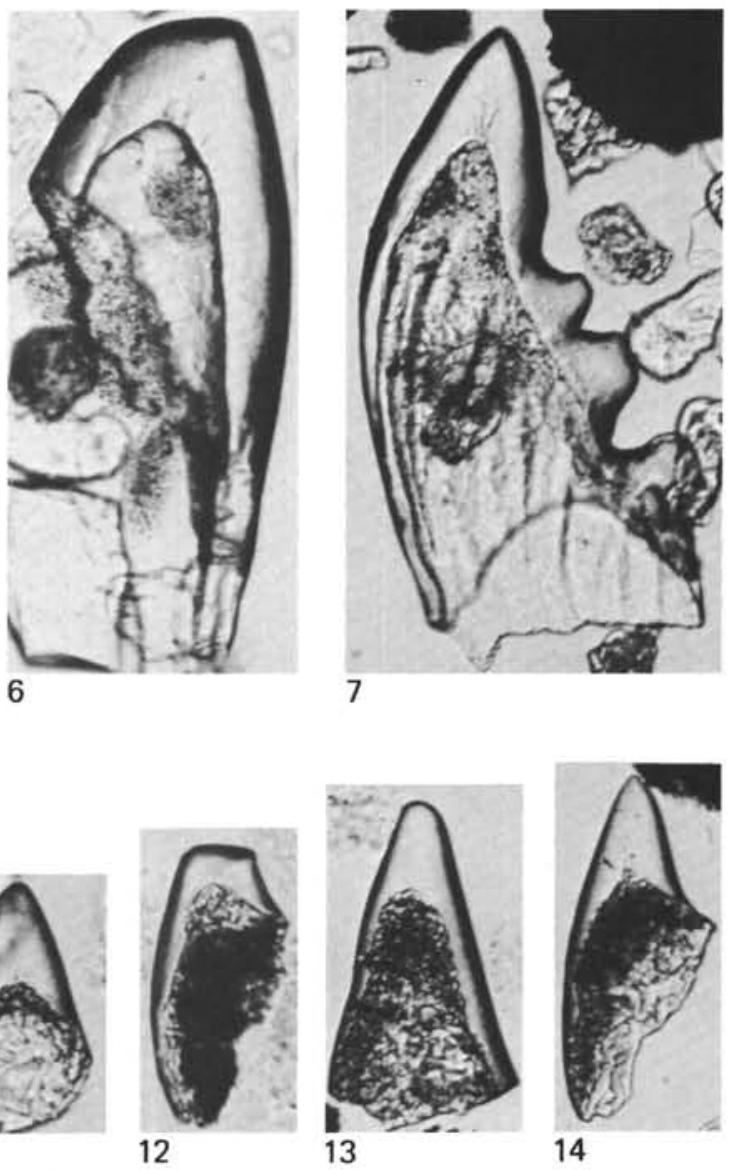

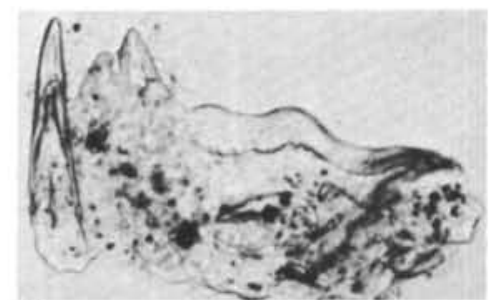

21
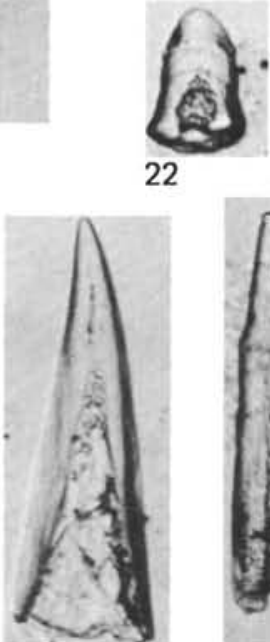

27

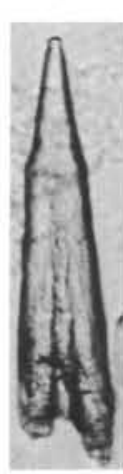

28

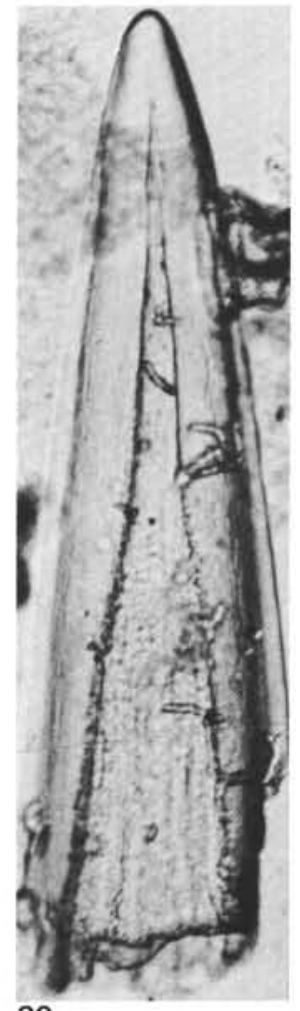

